\title{
Developmental Coordination Disorder and Cerebral Palsy: Is There a Continuum?
}

\author{
Jacqueline Williams • Christian Hyde • Alicia Spittle
}

Published online: 1 March 2014

(C) Springer International Publishing AG 2014

\begin{abstract}
Current diagnostic scheduling views cerebral palsy (CP) and developmental coordination disorder (DCD) as distinct disorders, though some hypothesise that the two lie on a continuum of motor impairment. We review recent evidence surrounding risk factors and underlying neurological mechanisms for both disorders, which we argue are central to clarifying this debate. Research highlights some common pre-, peri- and neo-natal risk factors for $\mathrm{CP}$ and $\mathrm{DCD}$, indicating potential similarities in aetiology. Neurophysiological data, however, are conflicting. There is evidence of an overlap in micro- and macro-structural abnormalities only when DCD
\end{abstract}

\section{J. Williams}

Institute of Sport, Exercise and Active Living \& College of Sport and

Exercise Science, Victoria University, Melbourne, Victoria, Australia

J. Williams · A. Spittle

Murdoch Childrens Research Institute, Melbourne, Victoria,

Australia

A. Spittle

e-mail: alicia.spittle@mcri.edu.au

C. Hyde

Cognitive Neuroscience Unit, School of Psychology,

Faculty of Health, Deakin University,

221 Burwood Hwy, Burwood, VIC 3125, Australia

e-mail: c.hyde@deakin.edu.au

\section{A. Spittle}

Department of Physiotherapy, University of Melbourne, Melbourne, Victoria, Australia

J. Williams $(\square)$

College of Sport and Exercise Science, Victoria University, Footscray Park Campus, PO Box 14428, Melbourne, VIC 8001, Australia

e-mail: jacqueline.williams@vu.edu.au

\section{A. Spittle}

Victorian Infant Brain Study, Murdoch Childrens Research Institute, Flemington Road, Parkville, VIC 3052, Australia samples include children exposed to pre- and peri-natal adversities, suggesting subgroups. There is a distinct lack of experimental work directly comparing children with DCD with and without exposure to pre- and peri-natal adversity, or children with $\mathrm{CP}$ and DCD. Hence, firm conclusions about the CP-DCD continuum debate cannot be drawn until neuroimaging and experimental work to this end is conducted.

Keywords Cerebral palsy $\cdot$ Developmental coordination disorder · Motor skill impairment · Preterm · Risk factors · Neural mechanisms

\section{Introduction}

Cerebral palsy (CP) and developmental coordination disorder (DCD) are disorders of movement that are most often considered to be separate entities, but at times, have been linked through terminology (minimal cerebral palsy is one of the past terms used to describe DCD [1]) or hypotheses that suggest that the two lie on a continuum [2]. A considerable evidence base has been established for the risk factors, causal pathways and neurological mechanisms for $\mathrm{CP}$, but far less has been understood in DCD and it is unclear what the relationship between the two is (if indeed there is any). While there is evidence that there are similarities in terms of the comorbidities that occur with each disorder [3•], and that perhaps deficits in higher order motor control processes such as movement planning and motor imagery may be similar in both populations [4-12], our understanding of whether CP and DCD are on a continuum rests on whether the underlying aetiology is similar. To this end, the most valuable information will come from studies on the risk factors and underlying neurological mechanisms for each disorder. In this paper, we review and compare the most recent risk factor studies for both $\mathrm{CP}$ and $\mathrm{DCD}$, and compare recent neuroimaging findings 
on DCD with the established evidence in $\mathrm{CP}$ to evaluate the hypothesis that the disorders lie on a continuum.

\section{Cerebral Palsy}

$\mathrm{CP}$ is an umbrella term to describe a group of disorders of the development of movement and posture, causing activity limitations, which are attributed to non-progressive disturbances that occurred in the developing foetal or infant brain [13]. CP is classified by the physical symptoms observed, based on the predominant type of motor disorder (spasticity, ataxia, dystonia or choreoathetosis) and the anatomic distribution. Spastic $\mathrm{CP}$ is characterised by hypertonic skeletal muscle most commonly resulting from lesions to upper motor neurons located in the primary motor cortex [14]. Ataxia most commonly results from damage to the cerebellum [15] and results in tremor, a lack of coordination and reduced balance. Dystonia and choreoathetosis are both forms of dyskinetic CP, most commonly resulting from damage to the basal ganglia [16]. These characteristics may be observed unilaterally, affecting one side of the body (hemiplegia), or bilaterally, involving either the lower body (diplegia) or all four limbs (quadriplegia), with significant variation in severity. Children with CP can have a range of functional abilities from walking independently to requiring full physical assistance for all activities $[17,18]$. These three factors (type, distribution and severity) combine to result in significant heterogeneity in motor impairment. In addition, the motor disorders of cerebral palsy are often accompanied by disturbances of sensation, perception, cognition, communication and behaviour, by epilepsy, and by secondary musculoskeletal problems [19].

\section{Developmental Coordination Disorder}

The predominant diagnostic features of DCD include a delay in motor development [Criterion A; Diagnostic and Statistical Manual, Fifth Edition (DSM-5); 20].

Like CP, DCD is characterised by significant heterogeneity, but unlike CP, reliable subgroupings based on clinical presentation are less clear. Studies continue to attempt to identify such subgroupings [21, 22], but consistent subgroupings across studies are difficult to identify. It is accepted that some children with DCD will experience only fine motor deficits, others only gross motor deficits and others a combination of both [23], but the characteristics of these impairments awkward, slow and less precise - appear to be similar regardless of the skill affected and there is currently no known aetiology that defines each subgroup.

Importantly, the diagnostic criteria for DCD have recently undergone subtle changes with the release of the DSM-5 [20]. Specifically, while onset early in the developmental period has long been a recognised feature of DCD, it is now acknowledged within the criteria (Criterion $C$ ). Furthermore, the final criterion (D) states that deficits in motor skills must not be better explained by intellectual disability, visual impairment or a neurological condition impacting movement (e.g. cerebral palsy, muscular dystrophy, degenerative disorder), which is now more specific than the related Criterion $\mathrm{C}$ in earlier iterations of the diagnostic criteria [24]. Previously, Criterion $\mathrm{C}$ stated that the disturbance in motor skills could not be the result of a "general medical condition" and this often resulted in questions over whether being born preterm precluded a diagnosis of DCD. Based on the new criteria, there is no reason that preterm birth should preclude a diagnosis of DCD, unless CP is diagnosed. This allows us to provide an interesting review of risk factors for DCD and their relationship to those for CP. Importantly, according to Criterion D, a diagnosis of CP precludes a diagnosis of DCD and implies that DCD itself does not have a neurological basis. However, this review will cite a number of recent studies that have identified atypical patterns of neural activation in DCD samples and discuss the relevance of this to Criterion D.

\section{Risk Factors for CP and DCD}

It has been suggested that CP and DCD may fall on a continuum on the basis that there is an overlap in pre- and peri-natal risk factors for each [2]. While this is often the case in the reported literature, a major limitation in older studies [25-27] and in those that we discuss below is that they do not compare differences in children with DCD born at term with those with children born preterm. Given that preterm birth is associated with a significant number of pre- and peri-natal adversities, it is unclear whether this produces a bias in findings. In CP, it has recently been reported that although many of the risk factors for CP in children born preterm are reported in those born at term, those born at term were more likely to have fewer of those same risk factors [28]. The authors suggest this may mean that those born at term were more likely to develop $\mathrm{CP}$ as a result of antenatal risk factors or the interaction of a smaller number of risk factors, whereas for those born preterm, CP likely develops from a multifaceted interaction of risk factors. That there are differences in the development of CP for children born term versus preterm may indicate the same would apply for DCD.

A small number of recent population cohort studies have reported on risk factors for DCD [29•, 30, 31], but only the study by Faebo Larsen et al. [29•] considered those born at term separately from those born preterm. The percentage of children born at term with DCD was $2.9 \%$, while $6.4 \%$ of children born preterm and $18.3 \%$ born very preterm were classified with DCD. Being born male and preterm were the biggest risks for DCD. Being small for gestational age was a risk factor in both term and preterm infants (and has previously been shown to increase the risk of CP [32]), while younger maternal age $(<25$ years) and increased maternal smoking 
were risk factors in term infants. While smoking is known to be a cause of restricted foetal growth, which is in turn a risk factor for $\mathrm{CP}$ [33], the finding that DCD risk increased with younger maternal age is in conflict with the commonly accepted CP risk factor of maternal age $>35$ years [34•]. There were no reported associations between maternal age and the other risk factors studied, so it is difficult to determine the cause of this finding and unfortunately the study did not include pre- and peri-natal risk factors more commonly observed in studies with preterm children.

Using an impressively stringent diagnostic protocol for DCD (notably lacking in most preterm studies), Lingam et al. [30] found that the risk ratio for DCD was significantly greater in those born preterm, with a birthweight less than $2500 \mathrm{~g}$ and in families with a lower socioeconomic status. Unfortunately, despite having an impressive number of children born at term and diagnosed with DCD in the study $(N=302)$, they did not consider risk factors for this group alone. Similarly, decreasing gestational age was demonstrated to significantly increase the risk of DCD when compared with children born at 40 weeks by Zhu et al. [31]. The risk of DCD was significantly increased at 37 weeks gestation, with an odds ratio of 1.5 , which increased to 4.8 at 31 weeks or less. This relationship with gestational age is confirmed by studies that restrict analysis to preterm samples, with reviews having previously demonstrated the increased occurrence of $\mathrm{DCD} /$ motor impairment in preterm children who do not develop CP $[35,36]$.

Although many more studies have reported on risk factors for DCD in preterm groups, there is no clear consensus on what factors are most likely to result in an outcome of DCD. Indeed, like $\mathrm{CP}$, it appears that the causal pathways are multifactorial and differ across children. In the most recent study, Zwicker et al. [37•] examined risk factors for DCD in children born with very low birthweight $(<1250 \mathrm{~g})$. There were a number of factors that differed between those in the sample with DCD and those without DCD, including gestational age and birthweight (both lower in DCD), gender (greater proportion of male individuals with DCD), as well as more common postnatal steroid exposure, longer duration of ventilation, more days on oxygen, retinopathy of prematurity and hyponatraemia. The authors took one further step and conducted a logistic regression to examine the relationship between these factors and movement assessment scores, finding that only birthweight, male sex and postnatal steroid exposure were significant predictors (gestational age was not included as a possible predictor because of its close relationship to birthweight). The effect of gender appeared to be independent of birthweight or gestational age, with no differences noted between male and female individuals on these factors. The authors argued that although low birthweight children with DCD were exposed to a number of neonatal risk factors to a greater extent than those without DCD, only one of these (postnatal steroid exposure) was actually a significant predictor of movement impairment. This is an important finding and indicates that the presence of a wide range of risk factors does not necessarily relate to the development of DCD.

The risk factors for $\mathrm{CP}$ are many and a thorough review has recently been provided [34•]. Many of these factors are commonly reported in relation to preterm births (e.g. multiple births, infections, male gender, maternal disease or infection, inflammation, stroke, seizures) and so it is not surprising that there is a significant overlap between risk factors reported in preterm DCD samples and CP samples. These risk factors also increase the risk of $\mathrm{CP}$ when present in term births. What remains to be examined is exactly how closely these risk factors are related to DCD in term-born children and whether the simple presence of these factors is actually related to motor outcome, with the Zwicker et al. study [37•] indicating that this is not always the case.

\section{Neural Mechanisms}

The neural mechanisms involved in DCD and CP are not fully understood and, like risk factors, in most cases are thought to be multifactorial [3•]. The human brain undergoes complex organisational changes during development both in and ex utero, with a range of both pathogenic and environmental events affecting how the brain develops [38]. Not surprisingly, there is more known about the neural mechanisms involved in $\mathrm{CP}$ than DCD, as the motor impairment in children with $\mathrm{CP}$ involves a disturbance to the developing brain. There has been a recent increase in the number of studies examining the neural mechanisms in DCD and we review here similarities and differences identified in DCD and $\mathrm{CP}$ at macrostructural, microstructural and functional levels.

\section{Macrostructural Mechanisms}

A recent systematic review identified that $80 \%$ of children with $\mathrm{CP}$ have some abnormal neuroradiological findings, with white matter being the most common. Interestingly, this leaves up to $20 \%$ of cases where no abnormality is detectable on conventional imaging [39], though this may be more likely in cases of mild or resolved CP [40]. There is a paucity of studies reporting on the presence or absence of macrostructural abnormalities in DCD samples, unless the sample is drawn from cohorts of children at an increased risk of perinatal adversities (e.g. preterm cohorts).

Brain development in children born very preterm (births $<32$ weeks of gestational age) provides a model for DCD and $\mathrm{CP}$ as it conforms to a spectrum of motor impairment severity with potentially common neural mechanisms. Amongst children born very preterm, between 30 and $50 \%$ may suffer specific early damage to peritrigonal brain regions of the cerebral white matter, known as periventricular leukomalacia 
(PVL) [41]. This is the dominant form of brain injury in children born preterm and is characterised by periventricular gliosis without tissue loss and secondary ventricular dilatation [42]. In individuals born preterm, there is a local focus and diffuse component to the injury, with the pathways that connect subcortical structures with cortical areas affected. This mostly results in motor impairments, however, cognitive and behavioural difficulties can also occur depending on the location of the injury [41-43]. As PVL involves a diffuse pattern of injury, both hemispheres are usually involved and, in the most severe cases of PVL, the motor impairment results in CP, with bilateral $\mathrm{CP}$ being the common presentation in children born preterm. However, white matter abnormality has also been identified in children born preterm with DCD. In a large study of 193 very preterm infants who had brain scans at term equivalent age, the severity of white matter abnormality related to the degree of motor impairment ranged from mild motor impairment to CP [44]. When children with CP were excluded from the analysis, there was still an association with motor impairment and white matter abnormality, suggesting that there is a neural component to all forms of motor impairment in preterm cohorts. This was also the conclusion of a recent review that determined that motor impairment in children born preterm was related to white matter abnormalities and severe magnetic resonance imaging abnormalities [45•]. The implications of these findings for children with DCD who were not at risk of perinatal adversity are unclear.

\section{Microstructural Mechanisms}

Diffusion tensor imaging was recently used in a small pilot study (DCD $n=7$; control $n=9$ ) to examine the integrity of motor, sensory and cerebellar pathways in children with DCD [46]. Both diffusivity and fractional anisotropy measures were reported for the corticospinal tract, posterior thalamic radiation and superior and middle cerebellar peduncles. Compared with a group of typically developing peers, the children with DCD showed no differences in fractional anisotropy in any area. This is in contrast to children with $\mathrm{CP}$, with a recent review reporting that decreased fractional anisotropy has repeatedly been found in the corticospinal tract in studies involving CP [47]. Zwicker et al. [46] also reported significantly reduced levels of mean diffusivity in the corticospinal tract in the DCD group compared with typically developing peers, with a trend towards lower diffusivity also noted in the posterior thalamic radiation, but no differences in the cerebellar peduncles. Again, this is in contrast to studies with $\mathrm{CP}$, with increased levels of mean diffusivity a regular finding in $\mathrm{CP}$ samples [47]. Interestingly, the findings relating to DCD are also in contrast to those of a very recent study that determined that very preterm children with a research diagnosis of DCD showed significant size reductions in fractional anisotropy of the right corticospinal tract compared with very preterm children without DCD and in both left and right tracts when compared with term controls [48]. The results of Zwicker et al. [46] may indicate that there are microstructural abnormalities present in DCD and that these abnormalities differ from those observed in children with $\mathrm{CP}$ and potentially also from those with DCD as a result of perinatal adversity. However, as noted, it is important to note that this was a pilot study with only seven children, two of whom had considerably lower axial diffusivity scores than the remaining five, which may have skewed the results. Replication is required and should also include information about any perinatal risk factors present in the sample.

\section{Functional Mechanisms}

Functional neuroimaging studies that examine motor control in $\mathrm{CP}$ are surprisingly lacking, though a similar paucity of functional studies has been noted for cognition, and in particular, executive function [49]. Because of the lack of research identifying structural differences in DCD, functional studies have been favoured, though still small in number. Researchers have identified higher order motor control processes in which deficits have been observed in DCD samples [see 50 for a recent review] and attempted to identify atypical neural activity underlying such deficits.

DCD functional magnetic resonance imaging study samples have been small and each study has used a different task (though typically variations of visuomotor tasks). An initial visuomotor tracking task identified reduced activation in the left superior and inferior parietal lobes and the left postcentral gyrus [51]. In contrast, during a trail-making task, another DCD group was found to show increased BOLD response across a greater number of brain regions than typically developing peers, spanning the frontal, parietal and temporal lobes, whereas the typically developing children activated more areas in the precuneus in the superior parietal lobule. [52]. Differing task demands (discrete vs. continuous) and differing amounts of practice pre-scanning may have contributed to the differences between these studies. In a follow-up study, Zwicker et al. [53] examined the effect of task learning on the same trail-making task and found that children with DCD showed lower percentage signal change across a number of brain regions compared with typically developing children. These included the dorsolateral prefrontal cortex, inferior parietal lobule and cerebellum, suggesting under-activation of the cerebellar-parietal and cerebellar-prefrontal networks. Interestingly, this profile of atypical neural function supports a recent meta-analysis of neuro-cognitive accounts of $\mathrm{DCD}$, suggesting that fundamental deficits generating and/or using predictive modes of movement and rhythmic coordination (both of which are subserved by dorsal systems and their associated projections) may account, at least in part, for the motor deficits typical of DCD [50]. While neuroimaging 
evidence directly challenging these accounts is scarce, recently, motor timing was examined, with participants required to respond to stimuli at predictable or unpredictable interstimulus intervals [54]. Typically developing children, in line with adult research, were found to increase activation in the dorsolateral prefrontal cortex, cerebellum and temporoparietal junction when responding to unpredictable versus predictable intervals. In contrast, the DCD group did not show such an increase and as a result, showed significantly less activation in these areas during the unpredictable sequences. In addition to these studies, a recent study using electroencephalography found that when planning and executing a simple visuomotor aiming task, young children with DCD show a reduced amplitude of movement-related cortical potentials compared with typically developing children, but that older children with DCD show a greater engagement of motor cortical resources [55]. The latter was deemed to be a compensatory strategy, with increased cortical recruitment required to overcome reduced ability.

Taken together, these findings suggest that the majority of children with CP have abnormalities in neural structure that can be identified using current neuroimaging methods. It also appears that many children with perinatal adversity who develop motor impairment (or DCD) show abnormalities in neural structure (though to a lesser extent than those who develop (P). In addition, the corticospinal tract development is compromised in both of these groups and this disruption is likely to extend to their afferent sensory pathways. What is unclear is what implications this has for the functional connectivity of the motor system during motor activity. In contrast, available data on structural abnormalities in DCD are quite limited. What does exist suggests no major structural deviations. This brings into question whether or not the motor impairment observed in children exposed to perinatal adversity can be considered the same as that experienced by children without such exposure, particularly in light of the conflicting findings around fibre tracts (though more research is required in this area). Furthermore, functional abnormalities in neural activation in DCD may point to the presence of a yet to be identified neural underpinning for DCD, which brings into question the DSM-5 Criterion D. This criterion should be carefully considered as neuroimaging work continues to emerge in the field. Again, more research is required before a conclusion can be drawn on a CP-DCD continuum.

\section{The Missing Pieces of the Puzzle}

The heterogeneity in DCD suggests that there are likely to be subtypes within the disorder and it is likely that each subtype is subserved by, at least partly, differing aetiologies. To shed further light on the CP-DCD continuum debate, it is therefore critical to identify subgroup/s of DCD that may be more likely to share a similar causal pathway and/or neurological aetiology with $\mathrm{CP}$. To do so, we believe the following are crucial:

1. Studies that examine the similarities and differences in risk factors, causal pathways, neural structure and function between children with $\mathrm{CP}$ and those at increased risk of pre- and peri-natal adversity, such as children born preterm. Given the significant overlap in risk factors, it is reasonable to expect that DCD present in children born preterm, if in fact a distinct subgroup from those born term (see point 2 below), would be the most likely form of DCD to fall on a continuum with CP.

2. Similar studies are required that compare DCD in samples of children who have experienced pre- and peri-natal adversity with those with DCD who have not. In the past, the DSM criterion that motor impairment was not due to a general medical condition [24] has led to debate about the inclusion of preterm children in DCD samples, but with the rephrasing of this criterion, there is no longer any reason based on current knowledge that this should continue. However, there does need to be an awareness that these two groups may indeed be distinct subgroups of DCD, which needs further exploration. In this vein, DCD researchers need to improve their reporting of the presence or exclusion of children born preterm in their samples.

3. Researchers, particularly working with preterm samples, need to apply the DSM-5 [20] diagnostic criteria for DCD more closely. Many studies with DCD (not preterm) claim to implicitly meet Criterion B as their sample has been drawn from a clinical population, indicating that the motor impairment observed likely impacts on daily living. It is important to note that the presence of motor impairment does not automatically allow for a diagnosis of DCD and the impact on daily living is rarely addressed in preterm studies. Indeed in this review, we have referred to studies that have used the term DCD without addressing this criterion or in the case of two studies, based their 'diagnosis' of DCD solely on a parent questionnaire [29•, 31]. Furthermore, it is important that intellectual disability and visual impairment are not contributing to the motor impairment (Criterion D). The use of the term DCD should not be used in future studies without appropriate diagnostic measures. If these are not met, another term, such as 'motor impaired', should be employed.

4. As noted earlier, both children with CP and DCD appear to share deficits in higher order motor cognition, including poor motor imagery and action planning [4-12], though no direct empirical comparison exists. While this overlap could be interpreted within the continuum framework of CP-DCD, we must be circumspect about doing so given the lack of supporting neurophysiological data. That is, while there are similarities in behavioural 
atypicalities, there is a lack of accompanying neurophysiological data that limits the degree to which the underlying causes (neural or otherwise) of poor performance, and whether they overlap for $\mathrm{CP}$ and $\mathrm{DCD}$, can be elucidated. Still, direct comparison of behavioural and neurological performance of those sub-types of DCD and CP (i.e. preterm) that are most likely to share a causal basis on tests of motor cognition may shed light on the underlying neuro-cognitive mechanisms that subserve both disorders, and how, if at all, they overlap.

\section{Conclusion}

Currently, there is limited evidence to either support or refute the presence of a continuum between CP and DCD. We suggest that it is possible there may be a subgroup/s of DCD that do fall on some form of continuum with $\mathrm{CP}$, but that this is most likely in children who were exposed to pre- and perinatal risk factors such as preterm birth. In the latter, there is some evidence that risk factors for both CP and DCD show considerable overlap and that the neural structure, at both macro and micro levels, shows similarities. However, there is a paucity of studies that directly compare children with $\mathrm{CP}$ and DCD or that compare children with DCD who were exposed to these risk factors with those who were not. Continued advances in neuroimaging, direct comparison of behavioural and neurophysiological outcomes on tasks of motor cognition and a greater number of population- and cohortbased studies will hopefully allow for more conclusive outcomes in coming years.

\section{Compliance with Ethics Guidelines}

Conflict of Interest Jacqueline Williams, Christian Hyde and Alicia Spittle declare that they have no conflict of interest.

Human and Animal Rights and Informed Consent This article does not contain any studies with human or animal subjects performed by any of the authors.

\section{References}

Papers of particular interest, published recently, have been highlighted as:

- Of importance

1. Sustersic B, Sustar K, Paro-Panjan D. General movements of preterm infants in relation to their motor competence between 5 and 6 years. Eur J Paediatr Neurol. 2012;16:724-9.
2. Pearsall-Jones JG, Piek JP, Levy F. Developmental coordination disorder and cerebral palsy: categories or a continuum? Hum Mov Sci. 2010;29:787-98.

3. Spittle AJ, Orton J. Cerebral palsy and developmental coordination disorder in children born preterm. Semin Fetal Neonatal Med. 2013. doi:10.1016/j.siny.2013.11.005. Motor impairments, including $D C D$ and $C P$, related to preterm birth are discussed in detail in the review. The prevalence, risk factors, comorbidities, assessment and intervention of $D C D$ and $C P$ in children born preterm are reviewed based upon current evidence.

4. Mutsaarts M, Steenbergen B, Bekkering H. Anticipatory planning of movement sequences in hemiparetic cerebral palsy. Mot Control. 2005;9:439-58.

5. Mutsaarts M, Steenbergen B, Bekkering H. Anticipatory planning deficits and context effects in hemiparetic cerebral palsy. Exp Brain Res. 2006;172:151-62.

6. Mutsaarts M, Steenbergen B, Bekkering H. Impaired motor imagery in right hemiparetic cerebral palsy. Neuropsychologia. 2007;45: 853-9.

7. Steenbergen B, Meulenbroek RGJ, Rosenbaum DA. Constraints on grip selection in hemiparetic cerebral palsy: effects of lesional side, end-point accuracy, and context. Cogn Brain Res. 2004;19:145-59.

8. Williams J, Anderson V, Reddihough DS, et al. A comparison of motor imagery performance in children with spastic hemiplegia and developmental coordination disorder. J Clin Exp Neuropsychol. 2011;33:272-82.

9. Williams J, Anderson V, Reid SM, Reddihough DS. Motor imagery of the unaffected hand in children with spastic hemiplegia. Dev Neuropsychol. 2012;37:84-97.

10. Williams J, Reid SM, Reddihough DS, Anderson V. Motor imagery ability of children with congenital hemiplegia: effect of lesion side and functional level. Res Dev Disabil. 2011;32:740-8.

11. Williams J, Thomas PR, Maruff P, Wilson PH. The link between motor impairment level and motor imagery ability in children with developmental coordination disorder. Hum Mov Sci. 2008;27:270-85.

12. Wilmut K, Byrne M. Grip selection for sequential movements in children and adults with and without developmental coordination disorder. Hum Mov Sci. 2013. doi:10.1016/j.humov.2013.07.015.

13. Rosenbaum P, Paneth N, Leviton A, et al. A report: the definition and classification of cerebral palsy April 2006. Dev Med Child Neurol. 2007;109 suppl 109:8-14.

14. Sheean G. The pathophysiology of spasticity. Eur J Neurol. 2002;9: 3-9.

15. Diener HC and Dichgans J: Pathophysiology of ataxia in humans. In: Plaitakis A, editor. Cerebellar Degenerations: Clinical Neurobiology. Norwell, MA, USA: Kluwer Academic Publishers; 1992. p. 261-80.

16. Himmelmann K, Hagberg G, Wiklund LM, et al. Dyskinetic cerebral palsy: a population-based study of children born between 1991 and 1998. Dev Med Child Neurol. 2007;49:246-51.

17. Ancel P-Y, Livinec F, Larroque B, et al. Cerebral palsy among very preterm children in relation to gestational age and neonatal ultrasound abnormalities: the EPIPAGE cohort study. Pediatrics. 2006; $117: 828-35$.

18. Vohr BR, Msall ME, Wilson D, et al. Spectrum of gross motor function in extremely low birth weight children with cerebral palsy at 18 months of age. Pediatrics. 2005;116:123-9.

19. Bax M, Goldstein M, Rosenbaum P, et al. Proposed definition and classification of cerebral palsy, April 2005. Dev Med Child Neurol. 2005;47:571-6.

20. APA. Diagnostic and Statistical Manual of Mental Disorders. 5th ed. Arlington, VA: American Psychiatric Publishing; 2013.

21. Pieters S, Roeyers H, Rosseel Y, et al. Identifying subtypes among children with developmental coordination disorder and mathematical learning disabilities, using model-based clustering. J Learn Disabil. 2013. doi:10.1177/0022219413491288. 
22. Vaivre-Douret L, Lalanne C, Ingster-Moati I, et al. Subtypes of developmental coordination disorder: research on their nature and etiology. Dev Neuropsychol. 2011;36:614-43.

23. Zwicker JG, Missiuna C, Harris SR, Boyd LA. Developmental coordination disorder: a review and update. Eur J Pediatr Neurol. 2012;16:573-81.

24. APA. Diagnostic and statistical manual of mental disorders. 4th ed. Washington, DC: American Psychiatric Association; 2000. text revised.

25. Landgren M, Kjellman B, Gillberg C. Attention deficit disorder with developmental coordination disorders. Arch Dis Child. 1998;79:207-12.

26. Pearsall-Jones JG, Piek JP, Rigoli D, et al. An investigation into etiological pathways of DCD and ADHD using a monozygotic twin design. Twin Res Hum Genet. 2009;12:381-91.

27. Pearsall-Jones JG, Piek JP, Martin NC, et al. A monozygotic twin design to investigate etiological factors for DCD and ADHD. J Pediatr Neurol. 2008;6:209-19.

28. Stoknes M, Andersen GL, Elkamil AI, et al. The effects of multiple pre- and perinatal risk factors on the occurrence of cerebral palsy: a Norwegian register based study. Eur J Paediatr Neurol. 2012;16:56-63.

29. Faebo Larsen R, Hvas Mortensen L, Martinussen T, Nybo Andersen A. Determinants of developmental coordination disorder in 7-year-old children: a study of children in the Danish National Birth Cohort. Dev Med Child Neurol. 2013;55:1016-22. The first and only study to consider pre and neo-natal risk factors for DCD separately for children born preterm and term.

30. Lingam R, Hunt L, Golding J, et al. Prevalence of developmental coordination disorder using the DSM-IV at 7 years of age: a UK population-based study. Pediatrics. 2009;123:e693-700.

31. Zhu JL, Olsen J, Olesen AW. Risk for developmental coordination disorder correlates with gestational age at birth. Paediatr Perinat Epidemiol. 2012;26:572-7.

32. Stoknes M, Andersen GL, Dahlseng MO, et al. Cerebral palsy and neonatal death in term singletons born small for gestational age. Pediatrics. 2012;130:e1629-35.

33. Dahlseng MO, Andersen GL, Irgens LM, et al. Risk of cerebral palsy in term-born singletons according to growth status at birth. Dev Med Child Neurol. 2014;56:53-8.

34. McIntyre S, Morgan C, Walker K, Novak I. Cerebral palsy: don't delay. Dev Disabil Res Rev. 2011;17:114-29. This comprehensive review of $C P$ includes discussion of classification, risk factors and causal pathways, with some discussion of differentiating motor impairments. The strength of the paper includes a review of the level of evidence for early assessment and interventions for children with $C P$.

35. Edwards J, Berube M, Erlandson K, et al. Developmental coordination disorder in school-aged children born very preterm and/or at very low birth weight: a systematic review. J Dev Behav Pediatr. 2011;32:678-87.

36. Williams J, Lee K, Anderson P. Prevalence of motor-skill impairment in preterm children who do not develop cerebral palsy: a systematic review. Dev Med Child Neurol. 2010;52:232-7.

37. Zwicker JG, Yoon SW, MacKay M, et al. Perinatal and neonatal predictors of developmental coordination disorder in very low birthweight children. Arch Dis Child. 2013;98:118-22. This study reported how several neo-natal variables commonly identified more frequently in DCD relative to control children were related to motor outcome. Once significant peri-natal risk factors were controlled for (i.e. low birth weight and male gender), only one neonatal factor (neonatal steroid exposure, a common predictor of poor motor outcome) significantly predicted motor competence.
38. Krageloh-Mann I, Horber V. The role of magnetic resonance imaging in elucidating the pathogenesis of cerebral palsy: a systematic review. Dev Med Child Neurol. 2007;49:144-51.

39. Korzeniewski SJ, Birbeck G, DeLano MC, et al. A systematic review of neuroimaging for cerebral palsy. J Child Neurol. 2008;23:216-27.

40. Wu YW, Croen LA, Shah SJ, et al. Cerebral palsy in a term population: risk factors and neuroimaging findings. Pediatrics. 2006;118:690-7.

41. Pavlova MA, Krageloh-Mann I. Limitations on the developing preterm brain: impact of periventricular white matter lesions on brain connectivity and cognition. Brain. 2013;136:998-1011.

42. Volpe JJ. Neurology of the Newborn Brain. 5th ed. Philadelphia, PA: W.B. Saunders; 2002.

43. Woodward LJ, Anderson PJ, Austin NC, et al. Neonatal MRI to predict neurodevelopmental outcomes in preterm infants. New Engl J Med. 2006;355:685-94

44. Spittle AJ, Cheong J, Doyle LW, et al. Neonatal white matter abnormality predicts childhood motor impairment in very preterm children. Dev Med Child Neurol. 2011;53:1000-6.

45. Peters LH, Maathuis CG, Hadders-Algra M. Neural correlates of developmental coordination disorder. Dev Med Child Neurol. 2013;55:59-64. Review of current neuroimaging studies of children with developmental coordination disorder and children with perinatal adversities and motor impairment (non-CP).

46. Zwicker JG, Missiuna C, Harris SR, Boyd LA. Developmental coordination disorder: a pilot diffusion tensor imaging study. Pediatr Neurol. 2012;46:162-7.

47. Scheck SM, Boyd RN, Rose SE. New insights into the pathology of white matter tracts in cerebral palsy from diffusion magnetic resonance imaging: a systematic review. Dev Med Child Neurol. 2012;54:684-96.

48. de Kieviet JF, Pouwels PJW, Lafeber HN, et al. A crucial role of altered fractional anisotropy in motor problems of very preterm children. Eur J Paediatr Neurol. 2013. doi:10.1016/j.ejpn.2013.09. 004 .

49. Weierink L, Vermeulen RJ, Boyd RN. Brain structure and executive functions in children with cerebral palsy: a systematic review. Res Dev Disabil. 2013;34:1678-88.

50. Wilson PH, Ruddock S, Smits-Engelsman B, et al. Understanding performance deficits in developmental coordination disorder: a meta-analysis of recent research. Dev Med Child Neurol. 2013;55:217-28.

51. Kashiwagi M, Iwaki S, Narumi Y, et al. Parietal dysfunction in developmental coordination disorder: a functional MRI study. Neuroreport. 2009;20:1319-24.

52. Zwicker JG, Missiuna C, Harris SR, Boyd LA. Brain activation of children with developmental coordination disorder is different than peers. Pediatrics. 2010;126:e678-686.

53. Zwicker JG, Missiuna C, Harris SR, Boyd LA. Brain activation associated with motor skill practice in children with developmental coordination disorder: an fMRI study. Int J Dev Neurosci. 2011;29: 145-52.

54. Debrabant J, Gheysen F, Caeyenberghs K, et al. Neural underpinnings of impaired predictive motor timing in children with developmental coordination disorder. Res Dev Disabil. 2013;34:147887.

55. Pangelinan MM, Hatfield BD, Clark JE. Differences in movementrelated cortical activation patterns underlying motor performance in children with and without developmental coordination disorder. J Neurophysiol. 2013;109:3041-50. 